\title{
Global SunFarm Data Acquisition Network, Energy CRADLE, and Time Series Analysis
}

\author{
Yang Hu, Mohammad A. Hosain, Tarun Jain, Yashwanth R. Gunapati, Lauren Elkin, G. Q. Zhang \\ and Roger H. French, Member, IEEE,
}

\begin{abstract}
Outdoor testing of photovoltaic (PV) modules plays a critical role in discovering the degradation modes, mechanisms and rates of materials, components and systems under real world climate conditions. The Solar Durability and Lifetime Extension (SDLE) center at Case Western Reserve University has established a highly instrumented outdoor test facility, the SDLE SunFarm. In order to study PV module performance under different climatic conditions, a global SunFarm network was established with nine PV outdoor test beds across the world. Energy CRADLE is an ontology driven database acquisition tool which is being developed to effectively store, analyze and query huge data sets generated by the SunFarms. The data collected from all these data sources will stream back to a MySQL database. A front end user interface was built for the purpose of data inquiry, equipment registration, maintenance, and metrology cross check. Design and characteristics of SunFarms are introduced. SunFarm informatics and two case studies will be discussed in this article.
\end{abstract}

Index Terms-photovoltaics, lifetime and degradation science, power degradation rate, time series analysis.

\section{INTRODUCTION}

A T the 2010 Department of Energy Science for Energy Technology workshop[1], the topic of photovoltaics(PV) lifetime and degradation science (L\&DS) was made a priority and its importance in the Mesoscale Science Report[2] was reconfirmed. A quantification of power decline over time, also known as degradation $\operatorname{rate}\left(R_{d}\right)$, is equally important as initial performance. As a fast developing industry, many new technologies are introduced by the PV industry every year. The prediction of lifetime and $R_{d}$ with new technology insertion requires a better understanding of the degradation mechanisms of PV systems, components and materials. A stress-response framework was developed[3] and applied to the indoor accelerated test of back surface acrylic mirrors[4]. This method can also be used for outdoor testing of PV to quantitatively determine its performance in the real world. Stressors in the real world including but not limited to solar irradiance, rain, snow, salt fog, and soiling. Real world exposure is always a combination of multiple stressors, which makes the test condition seem "uncontrolled". The attempt to isolate the influence of a single stressor or several stressors requires precision and redundant climate condition monitoring.

Yang $\mathrm{Hu}$, Mohammad A. Hosain and R.H. French are with the Solar Durability and Lifetime Extension center, Department of Material Science and Engineering, Case Western Reserve University, Cleveland OH, 44106 USA e-mail: (roger.french@case.edu).

Tarun Jain, Yashwanth R. Gunapati, Lauren Elkin and G.Q. Zhang are with the Department of Electrical Engineering and Computer Science, Case Western Reserve University.
On the other hand, these stressors can be simulated and duplicated with indoor accelerated test. With statistical and domain analytics[5] of indoor and outdoor testing, degradation mechanisms of PV technology can be ascertained.

Previous research on PV degradation rates focuses on getting more accurate and precise $R_{d}$ data[6][7][8][9]. Accurate and precise $R_{d}$ data need long observation times[10], normally 5-10 years. Additionally, in order to get a smooth trend, the impact of climate conditions is usually reduced by filtering out or averaging the raw data[11][12]. Our approach is to take continuous power data (minute by minute) and sufficient climate data monitoring, using spectra Time Series Analysis (TSA) to decompose the influence of climate condition on the power degradation.

\section{SUnFARM DESIGN \& CHARACTERISTICS}

\section{A. SDLE SunFarm}

The first outdoor test facility of SDLE Center SunFarm was established on the West campus of Case Western Reserve University. SDLE SunFarm is about one acre in size, divided into 16 individual electronic sites, including two sites of adjustable tilt rack and 14 high precision dual axis trackers. A total of 148 full-sized crystal silicon modules from 24 different manufactures in sets of six or eight were exposed on both tilt rack and trackers. Minute by minute semi-continuous monitoring of these modules provide time series data. On the trackers, 8000 samples will be exposed under 1X, 2X, 4X and $5 \mathrm{X}$ suns illumination with a front surface acrylic mirror concentrator. Spectral measurements can be taken on these samples monthly or bi-monthly to give point-in-time data.

Data monitoring systems of the SDLE SunFarm include power, insolation, and weather monitoring. For power monitoring, either inverters or I-V curve tracers were used. Two trackers with eight full-sized modules on each used Solectria PVI1800 string inverters. Another 10 trackers as well as two tilt rack sites used two brands of micro-inverters, Enphase and Powerone. On the other two trackers Daystar multi-tracer were used to take I-V curves of full-sized modules and minimodules with one minute time intervals. A portable I-V curve tracer was used on clear days to take I-V curves on demand.

Redundant insolation sensors were placed around the SDLE SunFarm in order to get accurate irradiance data and align the trackers. Four Kipp \& Zonen pyranometers of three different models (CMP6, CMP11, CMP21) were placed on the horizontal, tilt rack, and tracker planes. A Kipp \& Zonen pyrheliometer (CHP1) was used to measure direct illumination. An abedometer was used to measure the reflected 
irradiance from the ground. Multiple split-cell reference cells, Li-cor Li-200 pyranometers, and Apogee SP-212 full spectra radiance sensors were placed in the tracker plane to help align the tracker frames' orientations. Another four Apogee SP-212 full spectra irradiance sensors and apogee SU-100 UV sensors were mounted on the sample trays to measure the concentrated solar irradiance.

Two Vaisala WXD520 weather stations were placed on the SunFarm to record wind speed, wind direction, rainfall, rain intensity, rain duration, and humidity. An anemometer was connected to the Master Control Unit of trackers to monitor the wind load on the trackers. A snow cup was used to measure the precipitation. T-type thermocouples were used for backsheet temperature monitoring.

The data acquisition system consists of 17 networked Campbell Scientific CR-1000 dataloggers, with each datalogger connected to an AM 16-32 multiplexer, extending the capacity of datalogger to 32 differential measurement channels. The Campbell dataloggers monitor thermocouple and sensor outputs. Enphase micro-inverters use envoy unit to collect data from each individual micro-inverter. Similarly, Solectria string inverters use Solenview system to collect data. Minute by minute data can be downloaded from their web servers.

\section{B. SunFarm Network}

Cleveland's climate, a humid continental, is not typical for PV degradation research. In order to study PV modules performance under different climatic conditions, a global SunFarm network was established among nine PV outdoor test beds across the world. These test beds include four Ohio SunFarms: SDLE SunFarm, Cleveland, Ohio; Lakeview 1MW power plant, Cleveland, Ohio; Replex SunFarm, Mt. Vernon, Ohio; and AEP Dulan test center, Columbus, Ohio (Fig. 2).

Within the United States, we cooperate with two Q-Lab SunFarms in Arizona and Florida, which are in mid-latitude desert climate and humid subtropical climate area, respectively. On an even larger scale, we established three SunFarms abroad with international collaborators: Underwriter Lab Sunfarms in Taitung and Lijqu, Taiwan, and SunFarm at the Indian Institute of Technology, Gandhinagar (IITGN). These nine SunFarms span a large the range of environmental conditions across the globe. Similar data collection methods were applied to each SunFarm. In order to better manipulate the Big Data that streams back daily, and manage the sensors that go on each site, a data acquisition system Energy CRADLE was established.

\section{ENERGY CRADLE SUNFARM INFORMATICS}

The purpose of the Common Research Analytics and Data Lifecycle Environment (CRADLE) is to create for engineering, and in particular lifetime science, the tools and protocols necessary to transform Big Data to information, which informs scientific knowledge to guide further analysis[13][14][15][16]. Energy CRADLE is tightly focused on serving the needs of handling and sharing data among the SunFarm network. Fig 3 is a data flow chart of Energy CRADLE. Raw data collected from the SunFarms will go through data pre-processing and semantic annotation and stored in the database. With domain knowledge Energy CRADLE can manage the organization and orchestration of the data, making the inquiry of the data more efficient. The Energy CRADLE data integration environment has two features. First, it can push all the raw data collected from SunFarms on to a MySQL database. Second, it has a visual front end for data inquiry and sensor management.

The MySQL database schema was modeled on NREL's regional outdoor test facilities proposal. Applied to SunFarm data acquisition, each data source has a unique data format, so an individual table was built for each data source (Fig. 1). Raw data will be refactored into corresponding MySQL database table. A Map ID table was used to join different tables together. The front end of Energy CRADLE consists of four different web pages. According to their functions, the four pages were named: Data Inquiry, Equipment Registration, Maintenance, and Metrology Check. On the Data Inquiry page, data can be queried by location, system ID, local time, or local solar time. Invalid data points or NAs are filtered out. Equipment Registration page was built for the purpose of sensors and sample management. Location, serial number, and calibration coefficients can be registered remotely from any one of the SunFarms. Because the top of the tracker is more than 30 feet above the ground when it is operating, maintenance(e.g, changing samples) has to done when the track is at birdbath mode. Whenever maintenance is needed, the operator can go to the Maintenance page to specify the location and duration. Data collected during maintenance will be flagged in the database warning that the tracker is not at normal operation mode. The redundant insolation and weather sensors cross check to assure that sensors are working correctly. The Metrology Check page can plot the same variables collected by multiple sensors comparatively, making the cross checking easy.

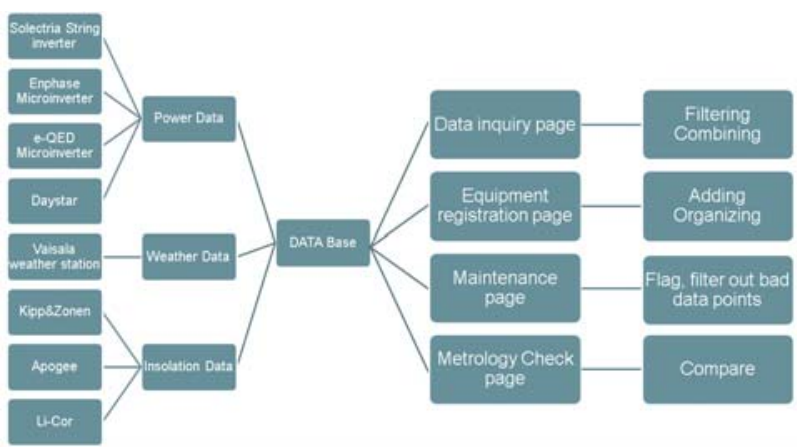

Fig. 1. Architecture of data acquisition system

\section{CAse Studies: Results}

\section{A. Mirror Augmented PV (MAPV) Performance}

A MAPV system and a non-augmented PV system's power production was recorded over 30 days, from February 19 to March 20, 2013. Seven non-augmented PV modules were connected into a string with a $30^{\circ}$ tilt. Another seven PV modules were tilted at $40^{\circ}$, with flat back surface acrylic mirrors tilted at $20^{\circ}$ towards the modules. The area of the 


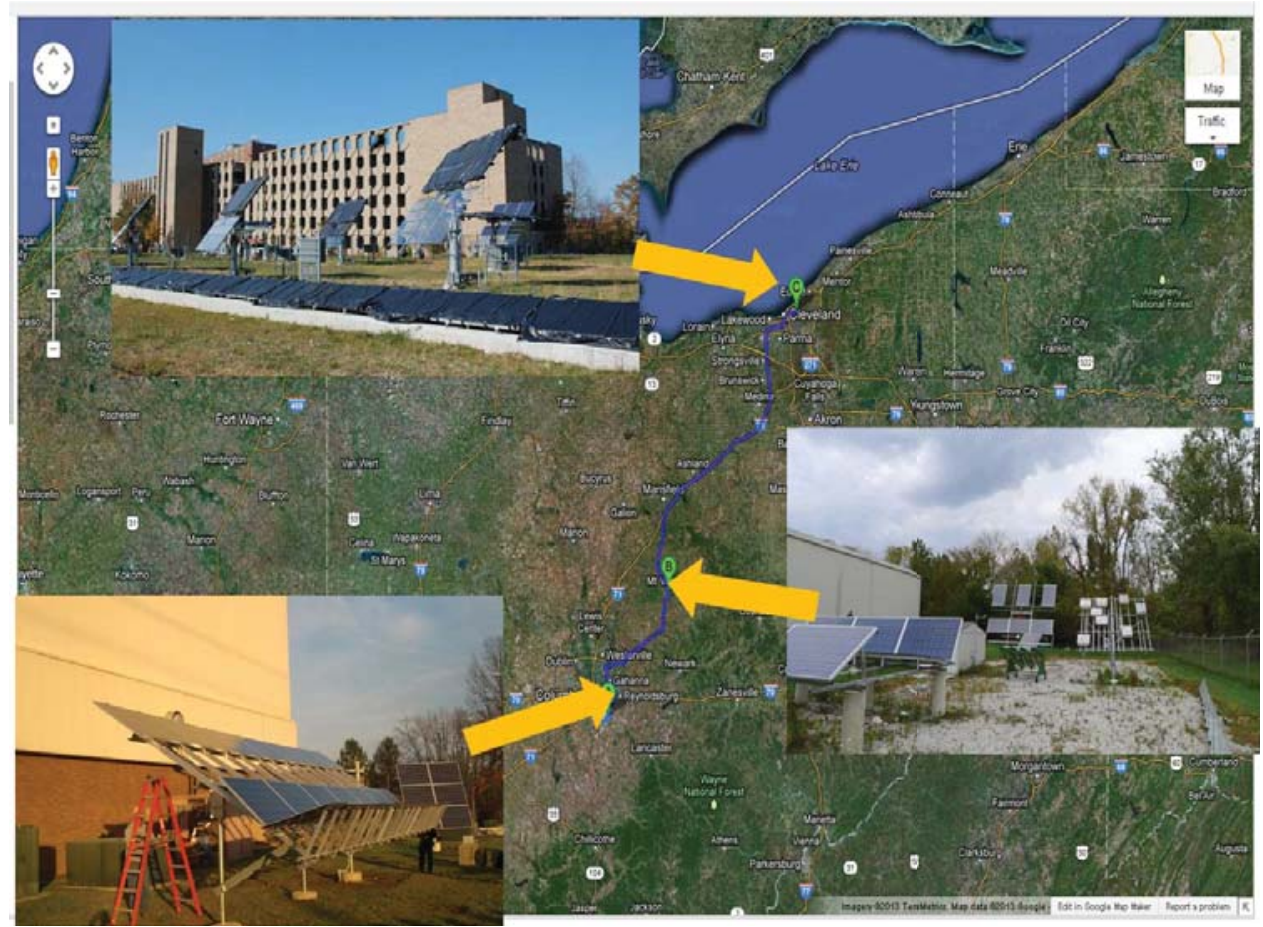

Fig. 2. SunFarms within Ohio. Upper left corner is SDLE SunFarm with tilt fixed rack in the front and duel axis trackers on the back. Bottom right is Replex SunFarm at Mount Vernon Replex Plastics, with fixed rack, single axis tracker and dual axis trackers. Bottom left is AEP SunFarm at Dolan Technology Center. Mirror Augmented PV (MAPV)system are on the bottom half of the tilt racks and flat back surface mirrors were mounted tilted towards the modules. The top half of the tilt rack has a non-augmented PV system.

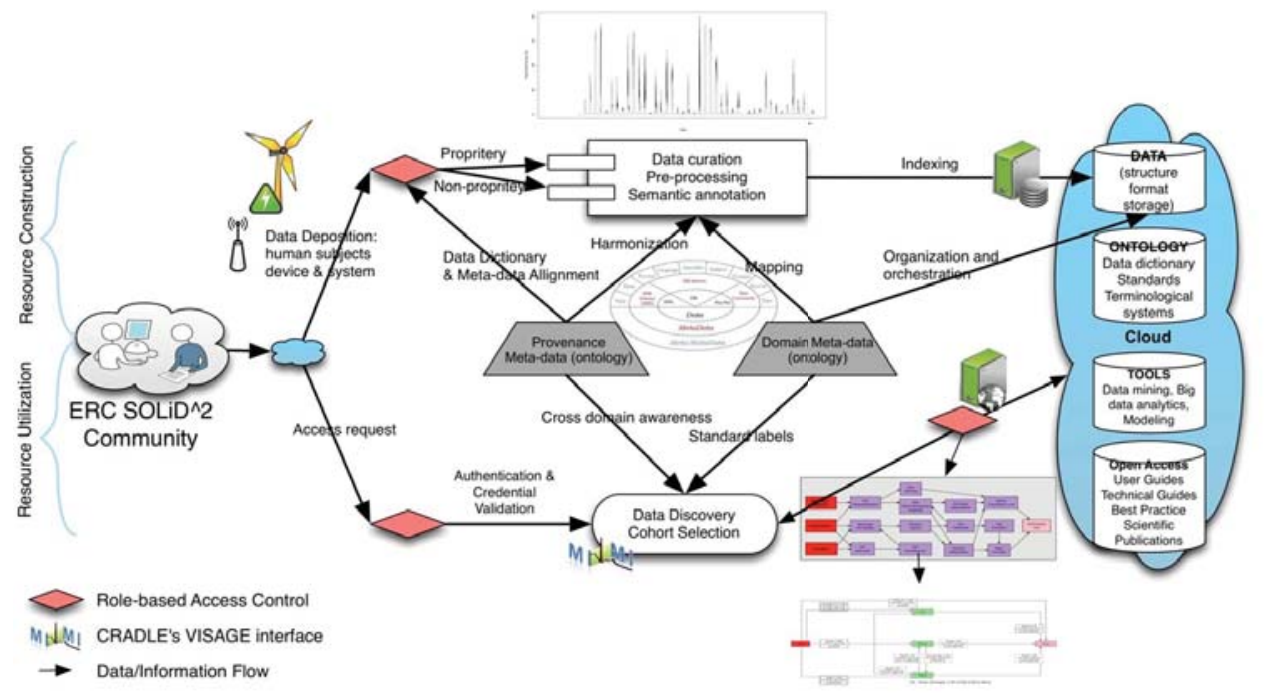

Fig. 3. Flow chart of Energy CRADLE

modules and mirrors are identical. Fig. 4 shows the power production of the non-augmented string and MAPV string from 9 a.m. to 4 p.m. on March 3nd, 2013. Fig.5 shows the power production of both the non-augmented string and the MAPV string over 30 days. Power production lower than $600 \mathrm{~W}$ was filtered out.
B. Cross sectional comparison of PV modules of 20 manufacturers

The power production of 60 crystal silicon PV modules from 20 different manufacturers exposed on the SDLE SunFarm was recorded by Enphase microinverters. Each brand has three duplicates. Among the population of 60,18 were on the fixed tilt rack, the other 42 were distributed on four trackers. In order to quantitatively compare the performance of each brand, performance ratio (PR) [17] was calculated from net energy output $E$, nameplate DC rating $P_{0}$, in-plane irradiance 


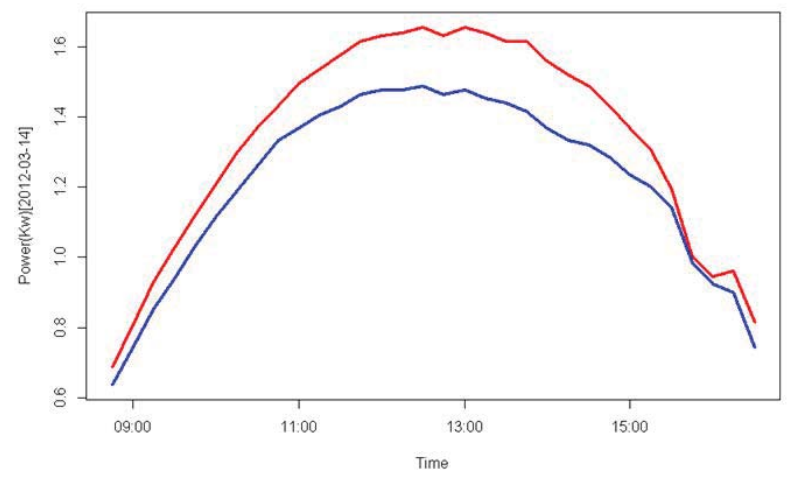

Fig. 4. Power production of seven non-mirror augmented PV modules in a string tilt at $30^{\circ}$ versus seven mirror augmented PV modules tilt at $40^{\circ}$ in one clear sunny day. The non-augmented string is shown in blue, the mirror augmented string in red.

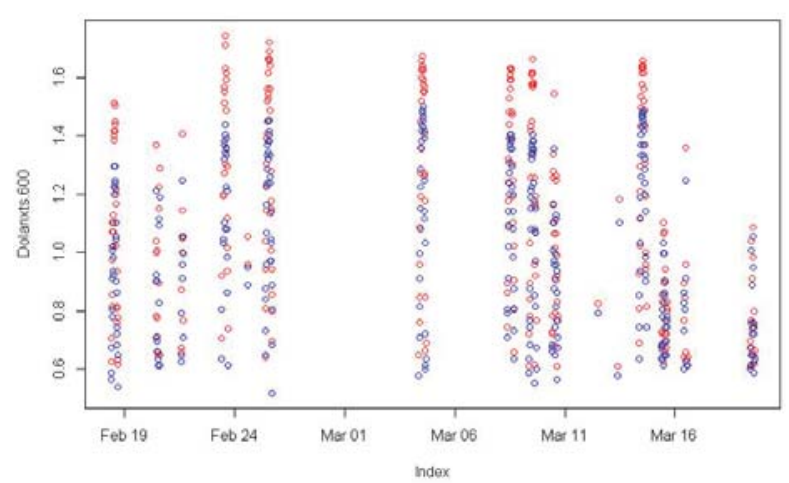

Fig. 5. Power production of both non-augmented and MAPV strings. A threshold of $600 \mathrm{~W}$ was applied.

$H$, and reference irradiation $G$ of each module. The relation of these variables is given by (Equation 1). $Y_{f}$ is final yield, $Y_{r}$ is reference yield.

$$
P R=\frac{Y_{f}}{Y_{r}} \text { where } Y_{f}=\frac{E}{P_{0}} Y_{r}=\frac{H}{G}
$$

Irradiance data was collected with a CMP 6 pyranometer set at horizontal plane and converted to in-plane irradiance of fixed tilt rack and trackers. Comparison was chosen at noon on a clear sunny day (Fig 6) to prevent the influence of clouds. Mean and standard deviation of 20 brands were plotted in Fig 7.

\section{CAse STUdies: Discussion}

\section{A. Mirror Augmented PV (MAPV) Performance}

In Fig 6 we observed a significant power boost (about $20 \%$ ) during the noon time of a clear day. Generally for a low concentration PV system, only direct solar radiance can contribute to the power boost. During cloudy days solar radiation was scattered thus low power boost was observed. The MAPV system tested at AEP SunFarm was designed to optimize its power production throughout the year[18]; its

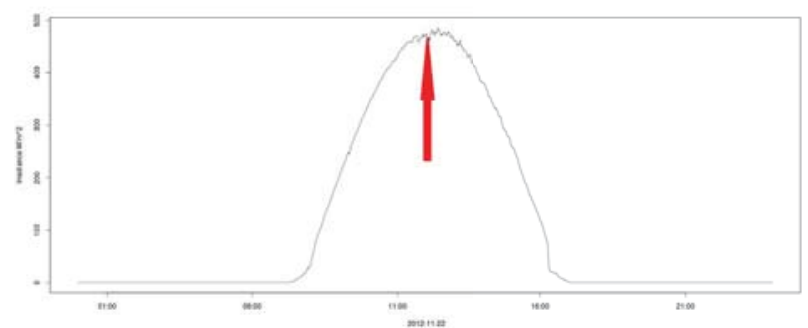

Fig. 6. Irradiance on horizontal plane on November 22nd, 2012. The dome shaped curve showing that it was a clear sunny day. The time when the cross section comparison was made is pointed with a red arrow in the figure.

noon-time power boost reaches the peak in March and October. Previous outdoor tests[19] shows 10\% annual noon-time power boost. In Fig.5 data collected on cloudy days was filtered out. The daily power boost in March is $5.8 \%$.

\section{B. Cross sectional comparison of PV modules of 20 manufac- turers}

The comparison of performance ratio get ride of differences of nameplate power rating, thereby reflecting the performance of each brand under the same climate condition. At the time this comparison was made tracker No.8 was under maintenance, and tracker No.12 was slightly off-tracking. So from Fig. 6 we observe a very low PR of brands $\mathrm{M}$ and $\mathrm{N}$, brands $\mathrm{O}$ and $\mathrm{P}$ showing large deviation. Otherwise, PR of most brands was above 0.8 and clear deviation can be observe among different brands, even at the same position (i.e, on fixed rack or on the same tracker).

\section{CONCLUSION}

On the SDLE Sunfarm, a continous automated minute by minute data acquisition system was implemented. Climate conditions including isolation, temperature, wind speed, wind direction, rainfall, rain duration, and snowfall were monitored. In order to test the performance of PV technologies under different climate conditions a SunFarm network was developed to sample a global selection of climate zones and conditions. To deal with the big data stream back from the SunFarm network, Energy CRADLE was proposed as a tool for data ensemble assembly and alignment.

\section{ACKNOWLEDGMENTS}

The authors acknowledge funding from Ohio Third Frontier, Photovoltaic Program Award Tech 11-060 and Wright Project Program Award Tech 12-004. The statistical analysis research was performed at the SDLE Center at Case Western Reserve University, funded through Underwriters' Laboratories.

\section{REFERENCES}

[1] J. Hemminger, G. Crabtree, and A. Malozemoff, "Science for energy technology: Strengthening the link between basic research and industry," A report from the Basic Energy Sciences Advisory Committee, US Department of Energy, 2010.

[2] J. Hemminger, "From quanta to the continuum: Opportunities for Mesoscale Science," A Report from the Basic Energy Sciences Advisory Committee, Tech. Rep., 2012. 


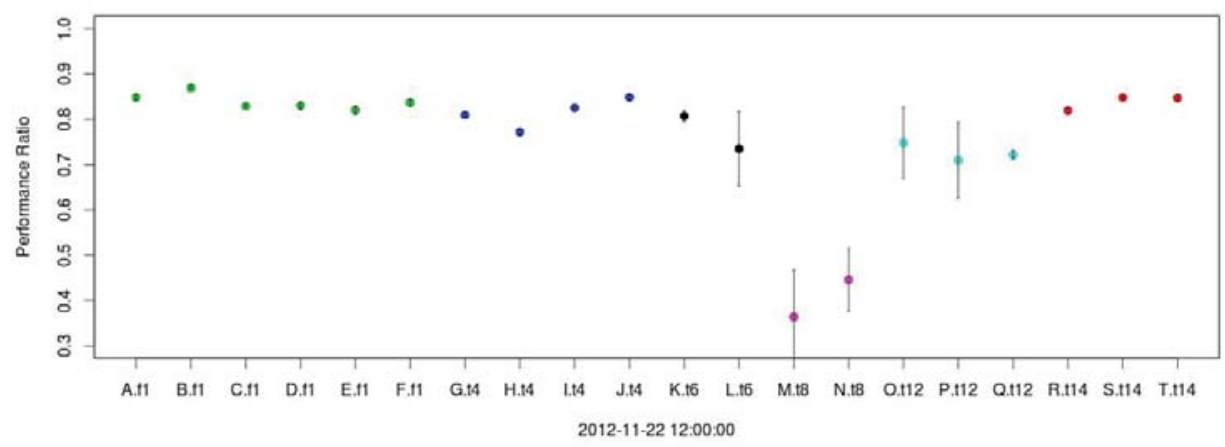

Fig. 7. Cross sectional comparison of crystalline silicon PV modules from 20 different manufacturers at noon November 22, 2012. Y axis is performance ratio range from 0.3 to 1.0. $\mathrm{X}$ axis shows the brands and location. Brand names were replaced with letters A through $\mathrm{T}$. Letter $\mathrm{f}$ or $\mathrm{t}$ are for fixed tilt rack and tracker. The performance ratio of three duplicates of each brand was measured. The dots show the average performance ratio of each brand. The standard deviation was plotted as error bar.

[3] M. P. Murray, L. S. Bruckman, and R. H. French, "Photodegradation in a stress and response framework: Poly(methyl methacrylate) for solar mirrors and lens," Journal of Photonics for Energy, vol. 2, no. 1, pp. 022004-022004, 2012. [Online]. Available: + http://dx.doi.org/10.1117/1.JPE.2.022004

[4] M. P. Murray, D. Gordon, S. A. Brown, W.-C. Lin, K. A. Shell, M. A. Schuetz, S. Fowler, J. Elman, and R. H. French, "Solar radiation durability framework applied to acrylic solar mirrors," Proc. SPIE 8112, Reliability of Photovoltaic Cells, Modules, Components, and Systems IV, 811203 (September 13, 2011), pp. 811 203-811 203-10, 2011. [Online]. Available: +http://dx.doi.org/10.1117/12.893827

[5] L. S. Bruckman, N. R. Wheeler, J. Ma, E. Wang, C. K. Wang, I. Chou, J. Sun, and R. H. French, "Statistical and domain analytics applied to PV module lifetime and degradation science," IEEE Open Access, Invited Paper, in press.

[6] D. Jordan, M. Kempe, D. Miller, C. Packard, J. Wohlgemuth, and S. Kurtz., "Observed field failures and reported degradation rates," in NREL Thin Film PV, February 2013.

[7] D. C. Jordan and S. R. Kurtz, "Photovoltaic degradation ratesan analytical review," Progress in Photovoltaics: Research and Applications, vol. 21, no. 1, pp. 12-29, 2013.

[8] D. C. Jordan, R. Smith, C. Osterwald, E. Gelak, and S. R. Kurtz, "Outdoor pv degradation comparison," in Photovoltaic Specialists Conference (PVSC), 2010 35th IEEE. IEEE, 2010, pp. 002 694-002697.

[9] R. M. Smith, D. C. Jordan, and S. R. Kurtz, "Outdoor pv module degradation of current-voltage parameters," in World Renewable Energy Forum, Denver, CO, USA, 2012.

[10] D. Jordan and S. Kurtz, "Photovoltaic degradation risk," in World Renewable Energy Forum, Colorado, 2012.

[11] D. C. Jordan and S. R. Kurtz, "Data filtering impact on PV degradation rates and uncertainty (poster)," in PV Module Reliability Workshop, 28 February - 2 March 2012, Golden, Colorado, 2012. [Online]. Available: http://nrelpubs.nrel.gov

[12] — , "The dark horse of," in PV Module Reliability Workshop, February 2627, 2013, Golden, Colorado, 2012. [Online]. Available: http://nrelpubs.nrel.gov

[13] G. Zhang, T. Siegler, P. Saxman, N. Sandberg, R. Mueller, N. Johnson, D. Hunscher, and S. Arabandi, "Visage: A query interface for clinical research," in Proceedings of the 2010 AMIA Clinical Research Informatics Summit; San Francisco. March 1213; 2010, March 2010, p. 7680.

[14] G. Zhang, S. Arabandi, and S. Redline, "Physio-MIMI lessons learned," National Center for Research Resources (NCRR), Tech. Rep., 2011.

[15] "Physio-MIMI homepage," 2012. [Online]. Available: http://physiomimi. case.edu/physiomimi/index.php/Main_Page

[16] R. Mueller, S. Sahoo, X. Dong, S. Redline, S. Arabandi, L. Luo, and G. Zhang, "Mapping multi-institution data sources to domain ontology for data federation: The PhysioMIMI approach," in AMIA Clinical Research Informatics Summit, March 2011.

[17] B. Marion, J. Adelstein, K. Boyle, H. Hayden, B. Hammond, T. Fletcher, D. Narang, A. Kimber, L. Mitchell, G. Rich et al., "Performance parameters for grid-connected pv systems," in Photovoltaic Specialists
Conference, 2005. Conference Record of the Thirty-first IEEE. IEEE, 2005, pp. 1601-1606.

[18] W.-C. Lin, D. Hollingshead, K. A. Shell, J. Karas, S. A. Brown, M. Schuetz, Y. Hu, and R. H. French, "Mirror-augmented photovoltaic designs and performance," in Energytech, 2012 IEEE. IEEE, 2012, pp. $1-5$.

[19] Y. Hu, D. Hollingshead, M. A. Hossain, M. Schuetz, and R. French, "Comparison of multi-crystalline silicon pv modules performance under augmented solar irradiation," in MRS Proceedings, vol. 1493. Cambridge Univ Press, 2013, pp. mrsf12-1493. 